\title{
THE CROP DISEASE AND PEST WARNING AND PREDICTION SYSTEM
}

\author{
Juhua Luo ${ }^{1,2}$, Wenjiang Huang ${ }^{1, *}$, Jihua Wang ${ }^{1}$, Chaoling Wei ${ }^{2}$ \\ ${ }^{1}$ National Engineering Research Center For Information Technology In Agriculture, Beijing, \\ P. R. China 100097 \\ ${ }^{2}$ Resources and Environment College of Anhui Agricultural University, Hefei ,Anhui \\ Province, P. R. China 230036 \\ * Corresponding author, Address: National Engineering Research Center for Information \\ Technology In Agriculture, Beijing 100097, P. R. China, Tel: +86-10-51503647, Fax: \\ +86-10-51503750,Email: yellowstar0618@163.com
}

\begin{abstract}
The aim of this study was to establish the warning and prediction system for crop diseases and pests based on SuperMap IS. NET geographic information system (GIS), which was developed by Supermap company. In this system, the author used GIS and remote sensing (RS) technology. The system could transform data information into a geographical information map to show the occurrence degree and distribution on various diseases and pests. This paper described mainly warning flow, database design and the main functions of the system. Finally, the system realized successfully the warning of the wheat stripe rust in Xifeng region of Qingyang city in Gansu province in 2002, and the prediction result was satisfactory. It indicated that we could classify and predict diseases and pests, and select right time and technology to control the diseases and pests by this GIS system.
\end{abstract}

Key words: disease and pest; geographic information system (GIS); Warning and prediction system

\section{INTRODUCTION}

The crop diseases and insect pests were all important biological hazard in agricultural production, and for many years, they restricted seriously the agricultural sustainable development. Statistics from the UN Food and

Please use the following format when citing this chapter:

Luo, J., Huang, W., Wang, J. and Wei, C., 2009, in IFIP International Federation for Information Processing, Volume 294, Computer and Computing Technologies in Agriculture II, Volume 2, eds. D. Li, Z. Chunjiang, (Boston: Springer), pp. 937-945. 
Agriculture Organization showed the world grain yield lost $10 \%$ because of pests and $14 \%$ because of diseases for many years. At the same time, the world cotton yield lost $16 \%$ because of pests and $14 \%$ because of diseases. China is a big agricultural country in the world, so the lose because of diseases and pests were approximately equivalent with the above statistic (Huang Muyi et al, 2003). It was more than important to predict the occurrence and development of diseases and insect pests by different prediction methods (Zeng Shimai,2005). According to prediction result, decision maker $\mathrm{s}$ and users could make correct prevention standards and proper treatment measures in order to obtain the maximum economic benefits on the condition of minimum capital investment .

The paper introduced a warning system of diseases and pests which was established based on GIS (geographic information system) and RS (remote sense). And the establishment of the system involved lots of objects, including agronomy, soil science and meteorology, and so on. Besides using the kinds of functions with GIS, the remote sense was also developed in the warning system to obtain fast and real-time the information of diseases and insects, which improved the accuracy of early warning result to a certain extent.

In summary, the establishment of the warning system of diseases and pests could provide scientific basis for and prediction scientific managing farm land.

\section{THE ESTABLISHMENT OF THE WARNING SYSTEM}

\subsection{The basic goals of the warning system}

The warning on diseases and pests was to predict the occurrence condition and the trend of development on diseases and pests in certain range and period, and made some strategy to control diseases and pests according to the pre-warning results ( Wang Shuhai et al, 2005).

The basic goals of the warning system were as follows:

1) By using the warning system, data information could be transformed into a geographical information map to show the occurrence and distribution on variety of diseases and pests by using visualization and spatial analysis with GIS.

2) The warning system could obtain fast and real-time the information of diseases and insects by using RS, and finally it could accurately monitor the occurrence and predict the development of the diseases and pests in large area (Jiang Jinbao et al, 2008). 
3) Some standards and measures could be made for the investigation and controlling diseases and pests according to the pre-warning results of the warning system.

\subsection{The design and realization the warning system of crops diseases and pests}

\subsubsection{Design route}

At first, it was important to choose feasible GIS platform and the prediction model on diseases and pests after requirement analysis. Second, database was established according to parameter of model, and function modules of the system were also designed. Finally, the warning system was established and published after debugging the system (Feng Jiangfan et al,2006).

\subsubsection{Platform selection}

SuperMap IS. NET was chosen as the system development platform, the reasons were as follows:

1) Components of design could be easily managed.

2) The Multi-source data could be integrated and the massive image could be quickly accessed to.

3) Server was clustering; it was with a high degree of flexibility

4) In the platform, Client and server belonged multi-level cache structure, which could support a variety of map engine work together.

\subsubsection{Database design}

\section{1) Attribute database}

The attribute database of the system was composed with monitoring data, basic data on diseases and pests, national meteorological observation data and the data table about latitude and longitude of meteorological observation site, and so on ( Wang Minghong et al,2006).

Monitoring data on diseases and pests: field investigation data, monitoring data using remote sensing, field experiments data, and so on.

Basic data on diseases and pests: diseases and pests species data, damage characteristics data, control and preventive methods data, and so on.

National meteorological observation data: temperature, moisture, rainfall and sunshine number, and so on.

\section{2) Spatial databases}

The spatial database of the warning was composed of basic map, thematic 
map and warning information map.

Basic map: national administrative division map, digital elevation map.

Thematic map was got by basic map according to some goal, which included weather map, remote sensing imagery and crop division map.

Warning information map was obtained by spatial analysis on the basic of few thematic maps and analysis result of prediction model, by which the system could show the occurrence and distribution on variety of diseases and pests. And ultimately, some effective guidance can be given to prevent and control the diseases and pests according the warning information map.

\subsubsection{Basic functions of the system}

The functions of warning system was composed of special functions and general functions which were offered by GIS platform, including the translating, mitigating and amplificating graphics, and adding and deleting map layer, and so on. While the special functions were as follows:

\section{1) Retrieval of diseases and insect pests}

Users could search all kinds of knowledge about diseases and pests, for example, latin name and English name of disease and pest, harm symptom and epidemic law on diseases and pests and control methods, and so on (Zhou Qiang,et al,2004).

\section{2) Diagnosis of diseases and pests}

Users could obtain some papers on warning results and prediction methods after the operations of systems by various data and prediction models of diseases and pests.

\section{3) Model base management}

Administrators could add, delete, or modify the models in the system by Model base management (Liu Shuhua et al, 2003).

\section{4) Monitoring and evaluation using remote sensing}

The function was realized by the module of pests monitoring. Multiple and multi-temporal remote data was applicated in the module, and assessment model based on RS was call in the module (SONG Y H et al,1993). By the function module, users could develop monitoring and assessment the diseases and pests. And related remote sensing image s could also were call from spatial database and displayed for users.

\section{5) Visualization of warning result}

By making visualization and spatial analysis with GIS, the system could transform prediction result and emergence grade into a geographical information map to display visually the occurrence and distribution on variety of diseases and pests. And ultimately, some effective guidance could be given by the system to prevent and control the diseases and pests (Si Lili et al, 2006). 


\subsubsection{Warning flow}

The warning system was established on the basic on a database which was composed of attribute database and spatial database and a model base including diseases models and pests prediction models. At first, the result which was obtained by prediction model form model base and data from database was displayed in the GIS platform and was transformed into information diagram, meanwhile, some thematic maps from spatial database were overlaid on the information map. Finally, the system could offer a clearly electronic information map which could show the occurrence and distribution on variety of diseases and pests (Fig.1).

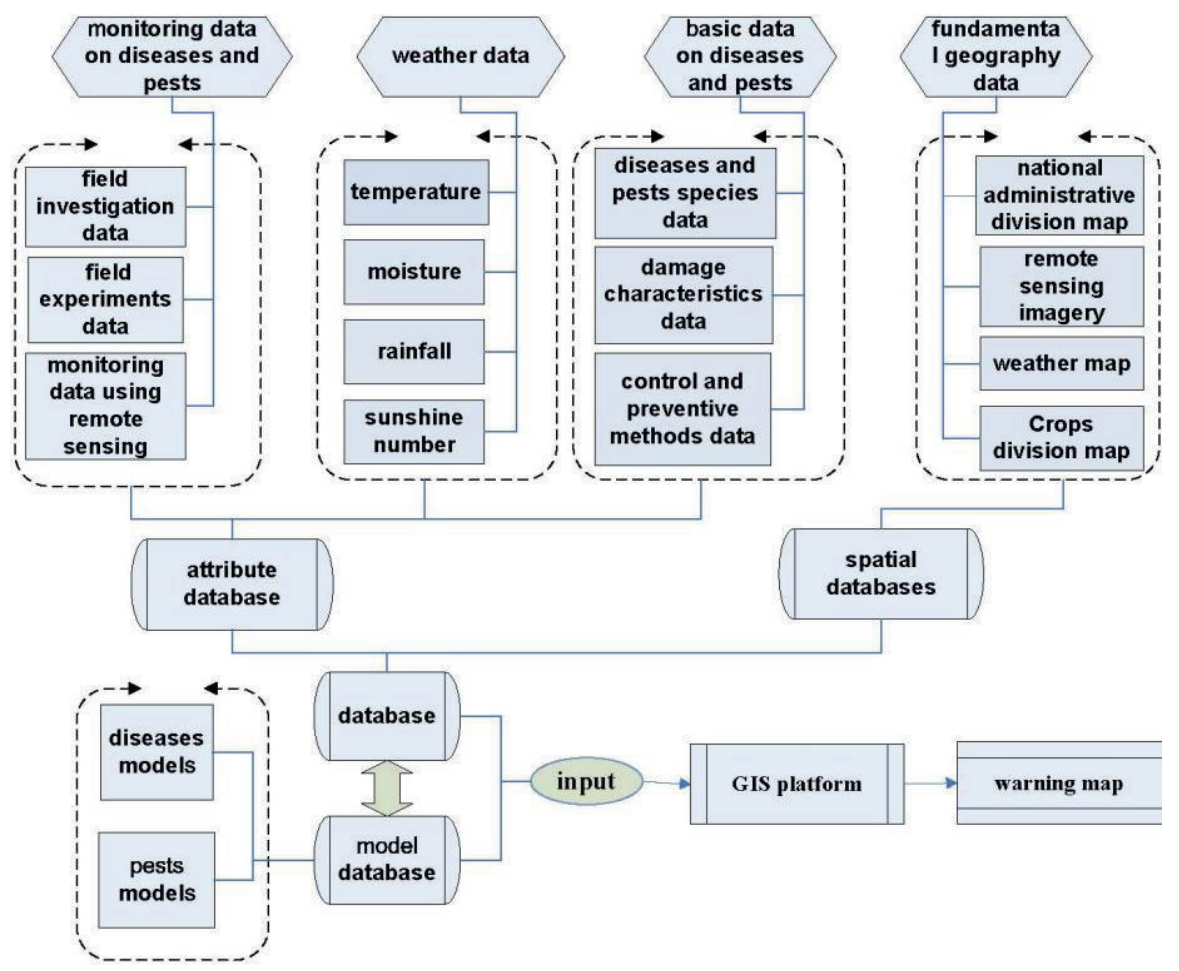

Fig.1. Flow chart of warning system for diseases and pests

\section{THE APPLICATION INSTANCE OF THE WARNING SYSTEM}

The process how to realize the function of the warning system was displayed by taking the wheat stripe rust disease in Xifeng of Qingyang region in Gansu province as an example. 


\subsection{Summary of study area}

The wheat stripe rust disease was one of the most serious diseases in China. Xifeng of Qingyang region in Gansu province was taken as an example to display the process how to realize the function of the warning system.

It was reported that the occurrence of the wheat stripe rust disease was mildly severe and severe symptoms in 1986,1989,1990,1991,2002,2003, outbreak in 1985, moderate in 1993, and mild or none occurrence in other years. The wheat stripe rust disease was intermittent disease, but the occurrence was continuous and frequent, and becoming more and more serious in Xifeng of Qingyang region in Gansu province in recent ten years.

\subsection{Data sources}

1) The data of wheat stripe rust disease was from plant protecting station in Qingyang region in Gansu province;

2) The weather data was offered by meteorological observing station in Qingyang region in Gansu province;

\subsection{Model selection}

At present, the models in the system were all from literature data. The model which was selected according to expert experience and model characteristics was as follows:

$$
y=0.3251+0.0414 x_{1}+0.0243 x_{2}+0.0667 x_{3}
$$

In above prediction model, $y$ is emergence grade, $x_{1}$ is average temperature from January to March, $x_{2}$ is index of temperature and rainfall, $x_{3}$ is index of the disease.

\subsection{Release the warning information according to the model}

\subsubsection{Grading standard on the stripe rust wheat}

Six degrees was adopted to evaluate the emergence of the wheat tripe rust, none, mild, lower than moderate, moderate, more than moderate, outbreak, and were correspondingly marked as $0,1,2,3,4,5$ and identified as white, blue, green, yellow, orange, red (table 1). 
Table 1 Grading standards for occurrence degree of wheat stripe rust disease

\begin{tabular}{cll}
\hline emergence grade & occurrence degree & warning color \\
\hline zone & none & white \\
one & mild & blue \\
two & lower than moderate & green \\
three & moderate & yellow \\
four & more than moderate & orange \\
five & outbreak & red \\
\hline
\end{tabular}

\subsubsection{The pre-warning results on study area}

\section{1) System interface}

The left page of the warning system concluded the field of user ' logging and operational area of prediction, while the data about the warning was display on the right of system interface with victor diagram.

\section{2) The operating process of the warning on the wheat stripe rust disease}

At first, the users could enter the main interface of the system by login; second, users could enter the main interface of diseases prediction by clicking the diseases prediction model. Finally, used could develop step-bystep operation in the operational area of prediction according.

\section{3) The pre-warning results on study area}

By the above operation, users could call, in the model from the database of the system, and got the warning result by calculation. Finally, the prewarning results were displayed on the right of the main interface by different color by electrical information map, at the same time, the warning results were also display by character form on the left of main interface (Fig.2).

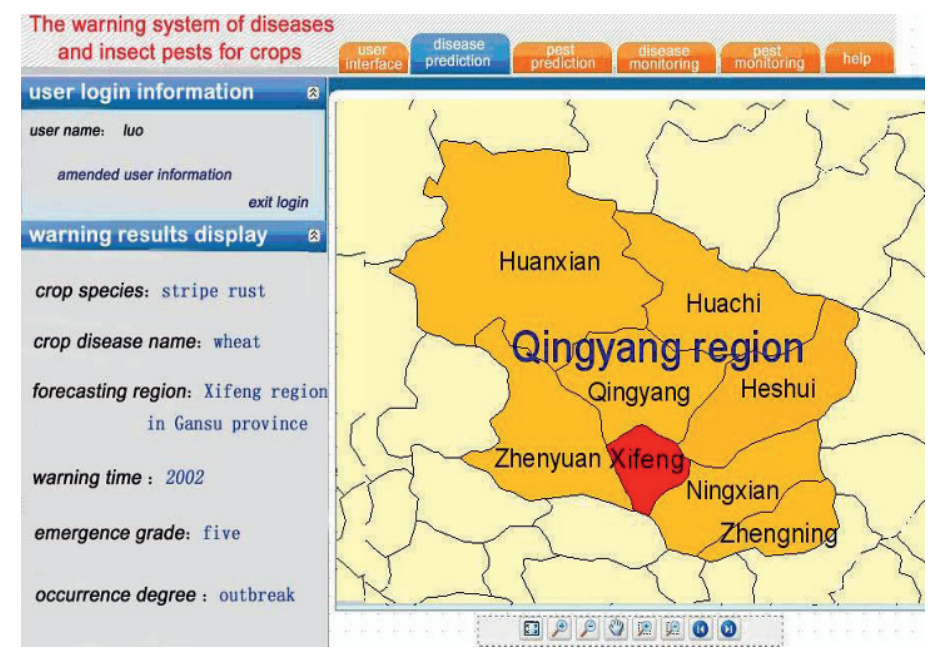

Fig.2. Pre-warning results of wheat stripe rust disease in Xifeng county of Qingyang city in 2002 
It was displayed that the emergence grade of the wheat stripe rust was five and occurrence degree was outbreak in Xifeng region of Gansu province. The fact that the warning results coincided well with report results from plant protecting station of Gansu province showed that this warning system and prediction method were all feasible.

\section{RESULT}

A warning and information system for diseases and pests of crops was established base on Geography Information System (GIS). The occurrence and damage of major diseases and pests of crops could be monitored and forecasted in real time by using the system, based on the forecasting model.And the system could submit warning maps with 6 colors and words information to the users. By using the system, we could standardize the forecasting data collection, transmit information through network and forecasting results viewable. Users could use it to classify and predict diseases and pests, also could use it to select fitting time and technology to control.

\section{DISCUSSION}

1) With the development of all kinds of subjects and lots of technology, many warning systems had been established at home and abroad. However, the system which was based on GIS was different from others traditional prediction system. The reasons were as follows: first, this system was based on .NET platform, and the interface was friendly, so the system was stable, portable, extensible and flexible. Second, this system could not only realize single point prediction but also regional prediction by basing on the function of GIS spatial analysis and multiple spatial interpolation methods. Finally, the remote sense was developed in the warning system to obtain fast and real-time the information of diseases and insects, which improved the accuracy of early warning result to a certain extent (BONE, S et al,2005).

2) The models in the warning system were all from literature data, so the models could only be used in some regions, which might result in the deviation of warning between theoretical value and actual value. However, with development and wide application of other information technology, the subsystem of automatic modeling could be established and used in the warning system to improve the accuracy of prediction. 


\section{ACKNOWLEDGEMENTS}

This work was subsidized by the National High Tech R\&D Program of China (2006AA10Z203, 2007AA10Z201), National Natural Science Foundation of China (40701119), and funds (2006BAD10A01, 2007BAH 12B02). The authors are grateful to Mr.Weiguo Li, and Mrs. Hong Chang for data collection.

\section{REFERENCES}

C. BONE, S. DRAGICEVIC and A. ROBERTS. Integrating high resolution remote sensing, GIS and fuzzy set theory for identifying susceptibility areas of forest insect infestations[J]. International Journal of Remote Sensing. 2005,26(21):4809-4828

Feng Jiangfan, Ying Xuewei, Zhang Hong, et al. Forewarning System of Blue Algae for Taihu Lake Based on GIS. Environmental Science \& Technology. 2006.29(9):59-62

Huang Muyi, Wang Jihua, et al.Hyperspectral character of stripe rust on winter wheat and monitoring by remote sensing. Journal of Agricultural Engineering Research.2003,19(6):154-158

Jiang Jinbao, Chen Yunhao, Huang Wenjiang, et al. Hyperspectral estimation models for LTN content of winter wheat canopy under stripe rust stress. Journal of Agricultural Engineering Research. 2008,24 (1): 34-39

Liu Shuhua. Yang Xiaohong, et al. Decision support system for crop diseases and insect pests prevention and control based on GIS. Journal of Agricultural Engineering Research 2003,19(4):148-150

Si Lili, Cao Keqiang, et al. Establishment of a real-time monitoring and forecasting system on main crop diseases and pests of China based on GIS. Journal of Plant Protection. 2006,33(3):282-286

SONG Y H,HEONG K L.Use of geographical Information system in analyzing large area distribution and dispersal of rice insects in South Korea[J].Journalof Applied Entomolite.1993,32(3):307-316

Wang Minghong, Jin Xiaohua, Liu Qian. The establishment of a long-distance warning and information system for major diseases and pests of corps in Beijing and its application. China Plant Protection. 2006,7(26):5-10

Wang Shuhai, et al. Establishment of space information database of prediction systems of Dendrolimus sp. Ased on GIS. Journal of Liaoning Forestry Science \& Technology. 2005,4(13) : 35-36

Zeng Shimai. Maco-phytopathology. Chinese Agricultural Technology Publishing House. 2005:182-184

Zhou Qiang, Zhang Runjie. Studies of W ebGIS-Based Warning Information System of Planthoppers Disaster. ACTA Universitatis Sunyatseni. 2004,30(21):64-66 